\title{
LOS POLÓMETROS: ACTITUDES LINGÜÍSTICAS SOBRE LA FONÉTICA Y LA MORFOLOGÍA DEL ESPAÑOL DE COSTA RICA
}

\author{
Polómetros: linguistic attitudes about the phonetics and morphology \\ of Costa Rican Spanish.
}

\author{
M. L. Alberto Barahona Novoa
}

Polo, -la adj. <<despectivo >> Campesino.// 2. Por extensión, mal educado, de mal gusto, rústico, que gusta de vestirse con ropa de colores chillones. (Voz usada en los centros urbanos con mayor frecuencia).// 3. Campesino que ha perdido los valores culturales que lo identifican con el agro. M.A Quesada Pacheco. (2007). Nuevo diccionario de costarriqueñismos.

\begin{abstract}
RESUMEN
En este artículo, se toma como objeto de estudio los llamados "polómetros", con el fin de analizar las actitudes lingüísticas que despiertan sobre la fonética y la morfología del español de Costa Rica. Estas actitudes construyen un perfil de hablante vitando, desprestigioso y excluyente que, sin ser el de un hablante de zonas rurales, se identifica con él. Se revela, entonces, la construcción ideológica en torno al cambio lingüístico del español costarricense.

Palabras clave: polómetro, español de Costa Rica, actitudes lingüísticas, ideologías lingüísticas, sociolingüística.
\end{abstract}

\begin{abstract}
In this article, the object of study is the so - called "polómetros", in order to analyze the linguistic attitudes that arouse on the phonetics and morphology of Costa Rican Spanish. These attitudes construct a discrediting and exclusionary speaker profile, which, although not a speaker of a rural area, is identified with him. It reveals, then, the ideological construction around the linguistic change of Costa Rican Spanish.

Palabras clave: Polómetro, Costa Rican Spanish, linguistic attitudes, linguistic ideologies, sociolinguistics.
\end{abstract}

\section{Introducción}

Es pertinente iniciar con un breve examen del epígrafe; pues en él, se encuentran las claves para entrever la tesis de este artículo. La primera acepción apunta al significado original de polo como adjetivo despectivo de campesino u hombre rústico. En la segunda acepción se recogen los significados creados por extensión: mal educado, de mal gusto, rústico, que gusta de vestirse con

\footnotetext{
${ }^{1}$ Universidad de Costa Rica. Profesor catedrático del Departamento de Lingüística de la Escuela de Filología, Lingüística y Literatura. Costa Rica.

Correo-e: jose.barahona@ucr.ac.cr

Recepción: 15-02-2017 Aceptación: 28-02-2017
} 
ropa de colores chillantes. Con este valor se registra de uso preferente en las zonas urbanas. Por último, en la tercera acepción se vuelve al campesino, pero aquel que ha perdido su identificación cultural con el agro; es decir, aquel que se ha desplazado -probablemente- a un entorno citadino, en el cual no se ajusta a los patrones de conducta allí estilados.

Esta entrada lexicográfica ofrece una enumeración de las características que configuran un tipo social. La primera es más restrictiva, pero las siguientes se orientan a la existencia de un prototipo social donde importan más las cualidades vitandas para la identificación de un polo que la referencia a un grupo social en concreto. Por tanto, se propone la existencia de un arquetipo social de rusticidad, falta de educación y mal gusto asociado al campesino, aunque no sea necesariamente una persona dedicada a las labores del campo.

Cabe preguntarse cómo surgen estas construcciones sociales que guían la conducta, actitudes, valoraciones y acciones de toda una comunidad. Existen muchas respuestas posibles: en este artículo se entorna la mirada hacia los polómetros, especie de test que permite que los integrantes de una sociedad puedan por sí mismos conocer cuál es su grado de "policidad"2. Estos formularios abordan distintos dominios de la vida cotidiana que reflejan esa condición, como: gustos musicales, tipos de bienes y servicios de consumo, costumbres, deportes, gastronomía, vestimenta, creencias, admiración por personajes públicos populares, uso de tecnologías, etc.

Específicamente en este artículo, se examinan detenidamente las referencias a los usos lingüísticos, considerados polos, no por su extracción campesina sino por esa construcción social urbana, que utiliza como punto de referencia al campesino y su forma de hablar, los cuales han sido tradicionalmente objeto de censura social. Podría decirse que se ofrece una descripción de un sociolecto que se asocia, aunque no exclusivamente, a clases sociales bajas o marginales. En consecuencia, los polómetros no se tratan de una valoración de la variedad sociolectal de los

\footnotetext{
${ }^{2}$ Acuño este neologismo para referirme al conjunto de valores, actitudes, gustos y comportamientos que articulan un tipo social vitando.
} 
campesinos, sino de la construcción de un prototipo lingüístico censurable, al igual como se han censurado las variedades lingüísticas rústicas.

En este estudio, se pasa examen a fenómenos lingüísticos de los niveles fonético y morfológico. También ofrecen información analizable sobre aspectos léxico-semánticos, datos relacionados con la onomástica, la valoración de las variedades lingüísticas y el componente notacional. Estos últimos temas serán materia para una publicación que amplíe el análisis de los polómetros.

\section{Aproximaciones teóricas afines}

El objeto de estudio de este artículo se configura con productos textuales que circulan en la Internet. Estos documentos que asumen una estructura de test o cuestionario tratan de constituirse en estímulos para que los integrantes de un determinado conglomerado social detecten el uso de costumbres, opiniones, creencias o formas de hablar, considerados o presumidos como desprestigiosos o excluyentes del grupo social hegemónico; así los destinatarios pueden saber en qué grado su comportamiento se acerca a ese modelo vitando. En especial, para este análisis interesan las intervenciones que se dirigen a recabar la información que se aporta concretamente sobre hechos lingüísticos.

Son, entonces, instrumentos para conocer las opiniones, los comentarios, los estereotipos o las ideas preconcebidas sobre el español de Costa Rica. Cabe resaltar que estas mediciones del grado de rusticidad de una persona se construyen anónimamente y por medio de una técnica que llamaríamos "en aluvión", pues a las versiones, año tras año, se les van agregando actualizaciones; de manera que se van formando grandes compilados de opiniones, aspectos, temas y estereotipos construidos alrededor de la variedad hispánica hablada en Costa Rica.

En el fondo de todo este fenómeno, queda claro que existe una consciencia sociolingüística del cambio lectal, el cual recibe una valoración positiva o negativa. En este caso, se establece un perfil vitando de actuación lingüística: el propio del hombre rústico, no urbano. Moreno Fernández 
(1998) afirma que esta consciencia es reflejo inequívoco de actitudes sicosociales; asimismo advierte que "si $[\ldots]$ las lenguas tienen un significado o unas connotaciones sociales, es natural que sean apreciadas y evaluadas de acuerdo con los estatus o las características sociales de sus usuarios.” (p. 80).

Puede, por tanto, colegirse que este artículo se basa en premisas propias de los estudios de actitudes lingüísticas. Se adopta como punto de partida, citando al mismo Moreno Fernández lo siguiente:

La actitud lingüística es una manifestación de la actitud social de los individuos, distinguida por centrarse y referirse específicamente tanto a la lengua como al uso que de ella se hace en sociedad, y al hablar de 'lengua' incluimos cualquier tipo de variedad lingüística hacia estilos diferentes, sociolectos diferentes, dialectos diferentes o lenguas naturales diferentes. (Moreno Fernández, 1998, p. 179-180)

Así las cosas, las actitudes lingüísticas mediante la valoración de los lectos entran en contacto con los usuarios de estos. De esta manera, se establece un vínculo con las identidades sociales y personales. Por estas razones, se puede concluir, en palabras de Moreno Fernández (1998), que "una variedad lingüística puede ser interpretada, por tanto, como un rasgo definidor de la identidad, de ahí que las actitudes hacia los grupos con una identidad determinada sean en parte actitudes hacia las variedades lingüísticas usadas en esos grupos y hacia los usuarios de tales variedades” (p. 180). Entonces, es comprensible que las actitudes mostradas en los polómetros hacia una variedad lingüística costarricense, a parte de enjuiciarla, construyen una identidad que, en este caso, se muestra desventajosa para el ascenso social y estigmatizadora.

Finalmente, no es ocioso advertir que este análisis deviene en un análisis de las ideologías que subyacen en la comunidad lingüística costarricense, puesto que se trata en definitiva del estudio de un producto cultural, bajo el entendido de que el lenguaje y sus diversas prácticas discursivas son una manifestación concreta de la cultura ${ }^{3}$.

\footnotetext{
${ }^{3}$ Esta aseveración se sustenta en las afirmaciones de Louis Althusser, citadas por Edmond Cros (2003) en su planteamiento de los estudios culturales, en especial sobre el sujeto cultural.
} 


\section{Antecedentes}

La comunidad de investigadores lingüísticos de Costa Rica ha incursionado, en forma exhaustiva y concienzuda, en el análisis de las actitudes lingüísticas desde varios marcos conceptuales y con alcances diferentes. También, es posible encontrar análisis, desde otras disciplinas sociales, que han seleccionado como objeto de estudio los polómetros. Por eso, se hace obligatorio tratar de sistematizar estos aportes de cara a la investigación que hoy se presenta. Por otra parte, una presentación exhaustiva de todos ellos sería ociosa, por lo que se reseñarán únicamente aquellos que, por su trascendencia, son indispensables de mencionar.

El punto de partida es, sin duda, el artículo titulado "Actitudes hacia el habla campesina de Costa Rica a través de la historia", escrito por Miguel Ángel Quesada Pacheco en 1989. En él, se desenmascara el "menosprecio del tico hacia sus propios valores: desprecio a su historia, a su música, a sus costumbres - calificadas como 'poladas'- y, por supuesto, a sus manifestaciones lingüísticas" (Quesada Pacheco, M. Á., 1989, p. 72-73). Esta cita es reveladora y retrata perfectamente los propósitos del autor: los usos lingüísticos costarricenses reciben una alta sanción social negativa. Se utiliza, para ello, el calificativo de "polada"; el profesor Quesada Pacheco presenta y examina su resemantización desde la referencia a lo rústico o campesino hasta su actual carga semántica relacionada a lo peyorativo o despreciativo; de manera que hoy polo no es campesino sino que "se utiliza para denotar a la persona de gustos no muy bien vistos por la población urbana, al 'mal educado'. " (Quesada Pacheco, M. Á., 1989, p. 81).

En fechas más recientes, C. Jara Murillo (2006) y A. Calvo Shaddid (2014) han publicado obras que dan cuenta exhaustiva de las actitudes lingüísticas hacia el español de Costa Rica e incluso de otras variedades dialectales hispanoamericanas. En "El español de Costa Rica según los ticos: un estudio de lingüística popular” (2006), Jara Murillo propone, desde la lingüística popular, cuatro modelos culturales construidos con las opiniones recabadas en 800 encuestas. A su entender, el modelo más extendido es el de reprobación absoluta, llamado "la nostalgia por el bien perdido", 
el cual está constituido por las opiniones más negativas. Le siguen en su orden: el modelo "sociolingüístico: la variación o diversidad como normal" que agrupa las opiniones más cercanas a la comunidad experta de sociolingüistas que considera el cambio como normal; luego, el modelo de "lealtad crítica: es bueno, pero...", en el que se sanciona negativamente el español costarricense, sin embargo presenta algunos errores; por último, distingue el modelo de máxima aprobación, el cual está formado por las manifestaciones de aprobación del dialecto costarricense, denominado "la lealtad condicional: aprobación absoluta y comparada". Cabe señalar que este último modelo recoge la menor cantidad de opiniones.

Por su parte, Calvo Shadid, en dos artículos publicados en 2014, presenta los resultados de una investigación que abarca no solo el estudio de las actitudes hacia el español de Costa Rica sino también considera otros aspectos. Específicamente, en el caso de Costa Rica, determina con cuáles variedades lingüísticas se identifican los hablantes de la zona metropolitana, indaga cómo evalúan otras variedades y analiza cuáles ideologías subyacen en sus actitudes. Asimismo, Calvo Shadid junto con Castillo Rivas amplía el análisis; pues no se limita a las variedades linguiísticas y sus actitudes vinculadas, sino que explora acerca de las opiniones sobre la corrección y la unidad lingüística, la valoración de otros dialectos hispanoamericanos, el uso del español en los medios de comunicación y el futuro de la lengua española.

La tesis de Laura Godínez para obtener el grado de licenciatura en Filología Española, sustentada en 2013, versa sobre las actitudes lingüísticas hacia el habla campesina, en este caso se circunscribe al cantón de Pérez Zeledón, por lo que sus alcances son más limitados que las obras antes mencionadas. Sus resultados coinciden con lo ya expuesto porque los hablantes de esa comunidad consideran que el habla campesina es incorrecta y que está llena de vicios, los cuales deben ser eliminados por la educación. Esta opinión predomina tanto en los jóvenes como en las mujeres, estas últimas se muestran como las que reprochan más acremente la deformación del idioma. Sin embargo, paradójicamente también se sostiene la posición contraria que exige que se 
conserve esta forma de hablar, pues se trata de la más auténtica y la que representa en forma ideal la idiosincrasia costarricense.

Desde la pragmática y los estudios culturales, Flores Cornejo (2012), analiza la palabra "polo" como medio que "sirve para excluir y desvirtuar lo que se sale de las normas de comportamiento o, por lo menos, lo que se considera normal" (Flores Cornejo, 2012, p. 108). En su explicación de cómo ese vocablo funciona como soporte ideológico en la creación de una identidad excluyente y centralista, revisa e interpreta su registro en las obras lexicográficas consagradas del español costarricense; además, destaca la importancia actual de los foros en medios electrónicos o “polómetros" en dicha tarea de construcción de identidades y otredades.

\section{El polómetro}

Las últimas tecnologías electrónicas y la red mundial de información Internet han permitido el surgimiento de plataformas que posibilitan nuevas interacciones discursivas, a saber "chat rooms", "blogs", perfiles en redes sociales, "tuits", etc. En todas estas modalidades, los participantes intervienen directamente y sus comentarios se elaboran con gran rapidez. Esta versatilidad admite incluso la escritura colaborativa y la participación simultánea de varias personas.

En este novedoso marco de escritura, han irrumpido los llamados polómetros que se estructuran a la manera de una encuesta y se difunden por estas plataformas y soportes comunicativos, con una rapidez increíble; así llegan a una multitud de destinatarios. Estas características los convierten en verdaderos mensajes virales.

El objetivo de estas encuestas es medir el grado de supuesta rusticidad de los participantes. Se ofrece una serie de preguntas sobre diferentes actitudes, gustos, actividades, etc., que pueden evidenciar cuál es el grado que se alcanza dentro de una categorización que se ofrece al final del instrumento. Los ámbitos escudriñados son, entre otros, las vestimentas, las actividades de entretenimiento, el arreglo personal, los gustos personales, la gastronomía, las costumbres, las 
creencias, la pertenencia a grupos sociales o étnicos, la admiración por algunos personajes públicos populares, el doninio del uso de nuevas tecnologías y el lenguaje. De esta forma, se construye un perfil prototípico del polo costarricense. Para mayor comprensión de los destinatarios, es frecuente que se reproduzcan situaciones concretas de la vida diaria, con el fin de que se seleccione su forma de reaccionar y así determinar si esta reacción o el empleo de cierto lenguaje se acerca a las conductas de una persona pola costarricense. Más adelante, se evidenciará por el análisis de estos componentes cómo este molde se aproxima sorprendentemente a la definición citada en el epígrafe. Asimismo, en este artículo, se enfocará la atención en los rasgos lingüísticos catalogados como propios de una persona pola; de hecho.

Es sabida la importancia que los estudios de actitudes lingüísticas conceden a la opinión de los usuarios acerca de una variedad lingüística. Para estas inquietudes heurísticas, los polómetros son excelentes fuentes de datos para dar cuenta de cómo los hablantes evalúan los hechos lingüísticos propios o ajenos. Se presentan en forma anónima e incluso su formulación no se reputa como de una sola persona. En la red social Facebook, existen páginas que formulan la pregunta ¿qué debería tener un polómetro en un determinado año? Ahí los seguidores de las páginas escriben sus aportes. Cabe resaltar, entonces, su naturaleza colectiva. Además, los polómetros se actualizan año tras año; se presentan por versiones o ediciones tal y como si fueran publicaciones periódicas ${ }^{4}$.

\begin{tabular}{|c|c|c|}
\hline Referencia bibliográfica & $\begin{array}{l}\text { Año de la } \\
\text { versión }\end{array}$ & $\begin{array}{l}\text { Código de } \\
\text { referencia para } \\
\text { las citas }\end{array}$ \\
\hline $\begin{array}{l}\text { Alvarado, Ó. (s.f.). www.angelfire.com. Recuperado de } \\
\text { http://www.angelfire.com/ky3/ozweb/polometro.html Consulta: } 26 \text { de julio de } \\
\text { 2015. }\end{array}$ & Sin fecha & Pol-sf \\
\hline $\begin{array}{l}\text { Chiri. (diciembre de 2004). www.basociacin4x4extremocostarica.runboard.com. } \\
\text { Recuperado de http://basociacin } 4 x 4 \text { extremocostarica.runboard.com/t1379 } \\
\text { Consulta: } 27 \text { de julio de } 2015 \text {. }\end{array}$ & 2005 & Pol-2005 \\
\hline $\begin{array}{l}\text { Romeovf. (30 de junio de 2008). www.forodecostarica.com. Recuperado de } \\
\text { http://www.forodecostarica.com/solaz/58039-polometro-2011-alguien-lo- } \\
\text { tiene.html Consulta: } 27 \text { de julio de } 2015 \text {. }\end{array}$ & 2008 & Pol-2008 \\
\hline $\begin{array}{l}\text { Anónimo. ( } 7 \text { de enero de 2010). www.ahweon.blogspot.com. Recuperado de } \\
\text { http://ahweon.blogspot.com/2010/01/polometro-2009-2010.html Consulta: } 27 \text { de } \\
\text { julio de } 2015 \text {. }\end{array}$ & $2009-2010$ & Pol-2009-2010 \\
\hline
\end{tabular}


En la recogida de datos, se logró encontrar las versiones de los siguientes años: $2005,2007^{5}, 2008^{6}$, 2009, 2010, 2012 y 2015; algunas carecen de fecha; así como una versión especializada para escritores ${ }^{7}$.

Las partes del polómetro reflejan las acepciones de la definición que sirve de epígrafe a este análisis. En primer lugar, la marca metalingüística $\langle<$ despectivo〉> se yergue como una supravaloración que domina toda la estructura del polómetro. Estos cuestionarios pretenden determinar, calificar y medir las conductas propias de la persona pola como modelo de comportamiento impropio, cómico, burlesco y, por tanto, vitando. Estas conductas generan que las personas desprecien o eviten caer en este prototipo social excluyente. Las puntuaciones obtenidas decidirán el grado de "policidad" que cada quien ostenta. Más adelante, se analizarán estas partes finales de los polómetros, pues en ellas abiertamente se redefine el concepto de "polo".

La segunda acepción de la definición se encuentra, ampliamente, ilustrada en estos documentos digitales. De hecho, muchos de ellos se encuentran segmentados por dominios muy cercanos a los que le sirvieron al Dr. Pacheco Quesada para organizar su definición lexicográfica. Se enlista una serie de acciones que revelan el grado de educación alcanzado. Se postula que los usos lingüísticos de este prototipo social son una forma de irrespeto a las normas aprendidas en el sistema escolar. Asimismo, se ofrece un amplio conjunto de actitudes o preferencias sociales que

\footnotetext{
Obando, A. (12 de octubre de 2009). www.elmasviolentoparaiso.blogspot.com.

Recuperado de http://elmasviolentoparaiso.blogspot.com/2009/10/polometro- $\quad 2009 \quad$ Pol-lit-2009 para-escritores.html Consulta: 27 de julio de 2015.

Anónimo. (2015). www.facebook.com. Recuperado de

https://www.facebook.com/puravidacrc/posts/326064557478504 Consulta: 27 de $2012 \quad$ Pol-2012 julio de 2015.

Rivera Villanueva, R. (5 de enero de 2015). www.medium.com. Recuperado de https://medium.com/@Rafalogo/polometro-2015-9f527b216b1a Consulta: 19 de mayo de 2015.

5 En esta versión del año 2007 no se incluyeron ítems lingüísticos. Su URL es la siguiente: http://www.forodecostarica.com/discusiones-del--debaul-los-recuerdos/354-polometro.html

${ }^{6}$ Del año 2008, se tiene una versión simple y una versión "reloaded", es decir recargada o especial. Esta última no figura en la bibliografía, pues se consiguió de un mensaje personal enviado por medio de correo electrónico. Esta versión "recargada" no aporta material nuevo de naturaleza lingüística, sino que en ella se agregan nuevas secciones de otros tópicos.

7 Este polómetro fue difundido en el año 2009 y se encuentra incluido en el blog que mantiene el escritor nacional Alexánder Obando, cuyo título es El más violento paraíso, homónimo de su novela. Por tanto, es el único con un autor conocido.
} 
dejan entrever cuán deplorable es su gusto. En muchas ocasiones, se hace referencia explícita a los campesinos como sujetos sociales portadores de conductas que calzan con el estereotipo del polo. El artículo de Flores Cornejo (2012), mencionado en los antecedentes, explota ampliamente esta veta de análisis sobre los sujetos culturales y la construcción de estereotipos.

\section{La valoración lingüística}

La estructura de los polómetros, en la mayoría de las versiones anuales, presenta una serie de actividades, gustos, costumbres o ideas que caracterizan o permiten identificar a una persona dentro de la categoría de polo dentro de la colectividad costarricense. Una de las áreas que sirve más a estos propósitos es la variedad lingüística de los individuos. La evaluación conduce a los participantes a identificar, en sus idiolectos, posibles rasgos o costumbres vitandas o bien sancionadas negativamente, a sabiendas de que quien las posea será candidato a recibir la etiqueta de polo.

En un primer acercamiento, se identificaron cuáles son esos elementos y, con ellos, estructurar una clasificación guiada por los niveles de la lengua, a saber: la fonética y la morfología. Sin embargo, luego de ese primer examen surgieron otros campos o dimensiones lingüísticas, como: el componente léxico de la onomástica, la valoración del dialecto hispánico costarricense dentro del diasistema español, la aceptación de giros lingüísticos costarricenses como normales y el componente notacional. Todos estos temas se presentarán en un artículo aparte.

\subsection{Fonética}

Algunas versiones destacan este componente como indicio de la condición de polo; de manera que su variación se convierte en prueba pertinente para la categorización sociolectal de los individuos.

Hay mención tanto de elementos segmentales como suprasegmentales; además, entre los primeros se puede distinguir entre consonánticos o vocálicos. Los polómetros advierten de su 
presencia, de los procesos del cambio lingüístico que los afectan; incluso se trasluce un intento de transcripción fonética.

\subsubsection{Cambios suprasegmentales}

En el polómetro codificado con la abreviatura Pol-sf, el cual carece de fecha, su autor quiso destacar una costumbre lingüística vitanda que la etiqueta como acento. Asimismo, en Pol-12, se encontró una variación de este mismo tema:

[1] 92. Cuando intenta usted comunicarse con un norteamericano. Usa acento "gringo" aunque lo que este [sic] hablando sea español? Pol-sf

[2] Doble puntaje si, así mismo, imita otros acentos latinoamericanos y se siente muy gracioso. Pol-sf

[3] Está aprendiendo a hablar inglés y hace los acentos del inglés en español? Es decir cuando habla con los compas intenta hablar como gringo? Pol-2012

Cabe destacar, en estos ejemplos, que la valoración no recae exactamente en el "acento" por sí mismo, si no en la función que este desempeña. En ambos casos, la sanción se relaciona con el vínculo que existe entre acento e identidad cultural lingüística. No obstante, también es posible advertir diferencias entre las afirmaciones. En [1] y [3] se sanciona la falta de lealtad con la identidad cultural propia, por la adopción de una costumbre enajenante. Además, la identificación con patrones culturales de los Estados Unidos se considera una práctica digna de censura social. Más adelante, se verá que esta misma valoración se esgrime para la adopción de anglicismos léxicos. Por su parte en [2], se descalifica el uso de otros acentos latinoamericanos para hacerse el gracioso. Entre ambas aseveraciones, puede notarse también que la segunda recibe una mayor sanción, ya que acredita doble puntaje para la determinación del grado de "polo" que poseen las personas. 
Se puede inferir, por estos ejemplos, que existe un alto grado de lealtad hacia la idiosincrasia lingüística de la variedad dialectal costarricense, al grado de considerar "polo" o de mala educación la imitación de otros acentos con fines ya sea de identificación a la cultura anglosajona o de desprecio de la cultura latinoamericana. Esta observación, se acerca a las consideraciones de Jara Murillo (2006) en su investigación sobre el español de Costa Rica; esta lingüista afirma que "la mayoría de los hablantes percibe la influencia extranjera como un factor negativo, no solo en relación con espCR ${ }^{8}$ sino con la cultura costarricense en general.” (p. 79).

\subsubsection{Cambios segmentales}

\subsubsection{Vocales}

En los polómetros analizados, el cambio evidenciado es la tendencia a la deshiatización de la secuencia -ea-, por medio del cierre vocálico de la vocal media y su conversión a un diptongo creciente o ascendente:

[4] Bailó “tiempo de vals” de Chayanne en sus "Quinciaños”? Pol-2005

En el ejemplo [4], la palabra "quinceaños" convertida en "quinciaños" acentúa irónicamente la consideración de cursi o ridículo de la acción de bailar una reconocida canción, a la cual se le pueden asignar tales calificativos. En este caso, el fenómeno lingüístico actúa como enfatizador o coadyuvante en la intención de censurar socialmente una costumbre.

En relación con este fenómeno, varios investigadores atestiguan su presencia en el dialecto hispánico-costarricense, por ejemplo Agüero Chaves (2009, p. 35), Calvo Shadid (2008) ${ }^{10}$ y Quesada Pacheco (1996, p. 543; 2002, p. 66) ${ }^{11}$. Aún más se puede afirmar que se trata de un rasgo

\footnotetext{
${ }^{8}$ EspCR es la abreviatura utilizada por Jara Murillo para referirse al español de Costa Rica.

${ }^{9}$ Cabe aquí recordar la definición de esta voz según Quesada Pacheco: Quinceaños m. Fiesta en la que se celebran los quince años de una joven (Nuevo Diccionario de Costarriqueñismos, 2007, p. 335).

${ }^{10}$ A diferencia de los otros autores, Calvo Shadid (2008) encuentra, en su estudio, algunos casos contrarios a la sinéresis, así apunta: "Adicionalmente, ha llamado la atención que en los manuales de dialectología se señale la tendencia general del español de América a la diptongación de los hiatos (sinéresis). Sin embargo, en una muestra tan pequeña como la estudiada, se han observado algunos casos de concurrencia de vocales en sílabas distintas que, si bien no marcan una tendencia, representan cierto porcentaje de separación de núcleos silábicos como corresponde a los hiatos." (Las semiconsonantes y semivocales en los diptongos del español: propuesta de análisis fonológico, 2008, p. 138).

${ }^{11}$ Esta lista de obras y autores no es exhaustiva, tan solo se citan los más significativos por difusión o especialización.
} 
general del español americano, ausente en la variedad paraguaya, según Manuel Alvar (citado por Quesada Pacheco, 2002, p. 66).

\subsubsection{Semiconsonantes}

La única referencia a cambios en los vocoides se encuentra en la versión 2012 del polómetro. Específicamente, se marca como fenómeno vitando las consonantizaciones que se pueden producir cuando la semiconsonante velar está en inicio de palabra. Así, "güeiso" (malo) derivado de hueso es parte del repertorio léxico considerado polo.

Este cambio ha sido citado por dialectólogos no solo para Costa Rica, sino para muchas partes de la geografía dialectal hispánica. Por ejemplo, en el Manual de dialectología hispánica ${ }^{12}$, Quesada Pacheco (1996) lo presenta como característica del español centroamericano. En nuestro país, Calvo Shadid (2008) ha analizado detalladamente este fenómeno; en sus resultados, no parece ser costumbre del habla culta del Área Metropolitana, excepto en la palabra huevo.

\subsubsection{Consonantes}

La asibilación del grupo biconsonántico - tr- es uno de los cambios mencionados en el corpus analizado, así como muchos investigadores lo han constatado para el español de Costa Rica, a saber: Calvo Shadid (1995, p. 132), Quesada Pacheco (1996, p. 554), Jara Murillo (2006, p. 50), Agüero (2009, p. 61) y muchos otros. Se transcriben especialmente los ítems obtenidos del polómetro del año 2012, pues estos se estructuran a la manera de una entrada propia de una obra lexicográfica con fines ilustrativos; es decir, se ofrecen ejemplos de uso como si se tratara de la recopilación de un corpus:

[5] Todas las palabras con "tr" las pronuncia como "trch". Por ejemplo, dice trchabajo en vez de trabajo, trchen en vez de tren? Pol-2012

[6] e) Trchompezó en vez de tropezó. Pol-2012

\footnotetext{
${ }^{12}$ Manual compilado y dirigido por Manuel Alvar. Esta obra goza de gran reconocimiento dentro de la comunidad de dialectólogos hispánicos.
} 
Resulta también muy interesante el intento de transcripción fonética popular de estas formas, claro está mediante grafemas que representan fonemas que, al parecer del lego en fonética, tienen algún grado de semejanza. En estos ejemplos, el recurso es la sustitución de la secuencia <tr> por la combinación <trch>. En última instancia, la intención es aprovechar el dígrafo <ch>, cuyo sonido representado corresponde a una consonante africada palatal sorda $/ \mathrm{t} /$; de esta manera, se aproxima perceptivamente al fenómeno de la asibilación. Este recurso grafemático popular también se presenta en la investigación de Jara Murillo (2006), cuya cita se reproduce más adelante. En este punto, vale recordar los resultados de una investigación llevada a cabo por Xinia Jaén sobre las actitudes de los hablantes guanacastecos hacia su propio dialecto, en la cual, estos refiriéndose a los hablantes vallecentraleños, se acude al recurso de representar la asibilación del grupo -tr- con el grafema $<\mathrm{ch}>$ :

Esto es absurdo: ellos, en San José, no pueden decir cuatro; no se entiende cuando hablan; casi dicen "guacho", ¿cómo hablando así puede ser uno más respetado? (Jaén, 1994, p. 73)

La articulación de la secuencia -tr- guarda un estrecho nexo con la asibilación de las vibrantes simple y múltiple, presente en la variedad hispánica de Costa Rica. Por tanto, sus valoraciones sociales corren la misma suerte. Carla Jara (2006) detectó que los hablantes nacionales sancionan negativamente la asibilación de las consonantes róticas, como lo apunta en la siguiente cita:

El aspecto más relevante para nuestro estudio en el área específica del nivel fonético, lo constituyen las referencias a sonidos específicos de espCR [español de Costa Rica] (15 referencias). Ya habíamos visto en la valoración negativa que la muy prominente característica que H [hablante] describe como 'arrastrar las rr' es señalada como un defecto de pronunciación o una causa de que la pronunciación sea 'mala'. En las referencias que se agrupan aquí, esta característica fonética nunca aparece positivamente señalada; encontramos señalamientos neutros como: la acentuada pronunciación de la rr lo distingue / el tico tiende a arrastrar la $r$. Pero con mayor frecuencia encontramos la valoración negativa del rasgo: 
mala pronunciación de la erre de ferrocarril, tres, matrícula / el habla popular (presenta) la distorsión en la silaba 'tr' al estilo inglés [chres] por tres. [cuachro] por cuatro / el costarricense no produce bien la rr / debe mejorar la pronunciación de aquellas palabras donde aparezcan la r, rr, tra, tre, tri, etc. / marcan demasiado la r y lo hace sonar muy feo / la ' $r$ ' la pronunciamos como con frenillo, no la pronunciamos correctamente y eso lo distinguen los extranjeros al primer momento. (Jara Murillo, 2006, p. 50-51)

En el polómetro del año 2008, figura una referencia a una articulación vitanda de otro fono. Sin embargo, la interpretación del fenómeno es azarosa, teniendo en cuenta tan solo dos hallazgos; por cuanto, se refiere a un sonido que aparece en un nombre propio de origen italiano: Versace, y en un préstamo del inglés. Gracias, también, a la aludida transcripción fonética popular en estos documentos, se representa la última sílaba de la palabra con el grafema <sh>:

[7] Le gusta la ropa de 'Versash'?. Pol-2008

En primera instancia, podría pensarse que se trata de la reproducción de un segmento fricativo postalveolar sordo $/ \mathrm{J} /$, el cual se ha registrado en variación libre con la africada palatal sorda /fg/ en regiones de la banda oriental del golfo de Nicoya, según Quesada Pacheco (1996, p. 551). Por tratarse, entonces, de una realización de carácter regional minoritaria, cabría pensar que la norma culta costarricense lo excluya y, posiblemente, sancione negativamente, de lo cual haría eco este comentario del polómetro 2008. Por otra parte, es viable considerar que se intentara tan solo recalcar la gran estridencia percibida, sin sugerir ningún fono vitando. En tercer lugar, tan solo podría ser la censura contra la incorporación de sonidos ajenos del español o el uso de xenismos, tal como ocurre con las referencias a la imitación de "acentos del inglés" y al uso de anglicismos. La falta de más información contextual en el ítem impide hacer una interpretación más certera de este rasgo fonético censurado.

Sorprendentemente, en el polómetro del 2009-2010, se vuelve a encontrar una representación grafemática <shh> relacionada a una palabra extranjera, como se constató en [7]:

[8] Les dice "joc dots" a los a los Hot Dogs y "sanguiches" a los sandwiches? Doble puntaje si les dice "sanguishh". Pol-2009-2010 
Para este ítem, valgan aquí las acotaciones apuntadas para [7]. Cabe resaltar, empero, la reduplicación de la hache, en el grafema <sh> para enfatizar la particularidad de la articulación de la última sílaba. Vuelve a aparecer esta rudimentaria transcripción fonética popular con el objeto de destacar quizás la estridencia del sonido. En ambos casos, el sonido sustituido es el representado por el dígrafo $<\mathrm{ch}>$.

Otro fenómeno fonético destacado es la alternancia entre la fricativa labiodental sorda /f/ y la fricativa glotal sorda /h/, como se aprecia en la siguiente oración obtenida del polómetro correspondiente al 2012:

[9] se jue eschilampao, en vez de se fue rápido. Pol-2012

Esta alternancia se ha reputado como rasgo propio del habla rústica, véase Agüero (2009, p. 50). En este aspecto, sí hay una coincidencia entre el modelo de habla vitanda propuesto en los polómetros y el habla de los pobladores de áreas no urbanas. A la vez, este rasgo se ha asociado a las variedades de las personas sin escolarización. Por estas razones, se evidencia que este rasgo del habla rural se asocia con la construcción ideológica del sujeto polo, realizada por los hablantes de entornos citadinos. Cobra aquí relevancia la observación de la segunda acepción de la palabra polo, que apunta Quesada Pacheco (2007, p. 322) en su diccionario, en el sentido de las relaciones entre lo rural y lo urbano.

En las observaciones fonéticas de los polómetros, no solo se hace referencia a cambios en la articulación de los sonidos, sino también se documentan procesos que los afectan como las neutralizaciones y elisiones. Ejemplos de estos tipos son los siguientes:

[10] No puede pronunciar la letra 'p' si se encuentra entre vocal y consonante. Ej: pecsi (Pepsi), pocs (Pops), excección (excepción), recección (recepción), calicso (calipso), excecto (excepto). Pol-s.f.

[11] se jue eschilampao, en vez de se fue rápido. Pol-2012

[12] pasa coyol quebrao, coyol comio (o está rajado), para decir que pasa limpio. Pol-2012

[13] Dice "compac dics" (CD's) en lugar de compact disc?. Pol-2008, Pol-2009-2010 
[14] Les dice "joc dots" a los a los Hot Dogs y "sanguiches" a los sandwiches? Doble puntaje si les dice "sanguishh". Pol-2009-2010

[15] A la catsup le dice “Capsu”? Pol-225 y Pol-2009-2010

En el ejemplo [10], se aborda el fenómeno de la neutralización de las consonantes oclusivas, en especial la alternancia entre el segmento bilabial sordo y el velar sordo, ante una consonante fricativa alveolar sorda /s/ en cinco de los seis ejemplos; tan solo en un caso se ilustra ante consonante oclusiva dental sorda /t/. Numerosas investigaciones han señalado la presencia de este rasgo en el español costarricense; asimismo, es menester recordar que la escolarización y la norma culta internacional del español lo han considerado una costumbre vitanda, propia de hablas poco prestigiosas.

Agüero Chaves (2009) se refiere a este cambio de las consonantes oclusivas sordas en posición heterosilábica. Sin embargo, según su parecer el sonido con el que alterna es un segmento fricativo bilabial sonoro [ß]: "lapso [láp.so, láb.so, láb̨.so]” (2009, p. 46); para el caso de la secuencia pt, documenta la pérdida del segundo segmento. No obstante, en Pol-s.f., se percibe el cambio como una neutralización de oclusivas, en consonancia con los datos ofrecidos por Quesada Pacheco (1996), quien afirma que, entre las formas en que cambian estas consonantes, se encuentran las sonorizaciones, “o bien, neutralización: [ıeфlegsjo'nar] reflexionar, ['segto] recto, [epi'legtikos] epilépticos" (1996, p. 543).

De acuerdo con la información recopilada en [11] y [12], otra característica vitanda es la elisión de las consonantes oclusivas sonoras en posición intervocálica, sobre todo en los participios perfectos en función de adjetivos como: quebrao < quebrado, comío < comido y eschilampao < deschilampado. Es curioso que no se aplica el mismo cambio en la forma "rajado", aunque el entorno lo favorecería, quizá por tratarse de una glosa que el autor prefirió transcribir con la forma canónica, ya que es la escritura que conviene al tipo de comentario en el que se inserta. 
Quesada Pacheco (1996) presenta este cambio y cita estudios específicos sobre este fenómeno, a saber: J. A. Fernández (1982) y F. López $(1987)^{13}$. Como se puede apreciar, es un rasgo ampliamente documentado en los estudios dialectológicos nacionales, e incluso Fernández afirma que se extiende por América Central, Colombia, Bolivia y Argentina, y algunas partes de México y el Caribe.

En cuanto al adjetivo eschilampao, es oportuno destacar que el proceso de aféresis de la dinicial se considera una característica censurable en las costumbres lingüísticas de los costarricenses. De hecho, Quesada Pacheco, en su diccionario de costarriqueñismos, recoge esta voz en su forma "eschilampado"; pero para su definición la remite a deschilampado. Ya se sabe que, en esta técnica de definir por remisión, subyace una calificación de preferencia por una de las formas en cuestión.

Las metátesis consonánticas también son objeto de consideración en los polómetros, como sucede en [13], donde "disc" pasa a "dics", tal vez como una forma de conservar la información morfemática de número, en préstamos lingüísticos del inglés; por lo que este ejemplo puede sumarse a otros donde se condena la mezcla de códigos, sobre todo con el inglés.

En [14], se presentan otras neutralizaciones (joc dots < hot dogs), pero interesa destacar ahora la sustitución de sandwiches por sanguiches. Este cambio obviamente influenciado por la presencia de la aproximante, labiovelar, sonora /w/, que provoca una asimilación en el punto articulatorio de la oclusiva, dental, sonora /d/ que pasa a ser velar, sonora $/ \mathrm{g} /{ }^{14}$. La alternancia entre estas oclusivas, aunque a la inversa, ya había sido registrada por Agüero Chaves (2009, p. 49) para las formas sanguijuela > sandijuela.

La supresión de sonidos al final de vocablos o apócope también encuentra lugar entre el elenco de fenómenos vitandos en relación con los segmentos consonánticos. En [15] aparte de la

\footnotetext{
${ }^{13}$ Las referencias completas de estas obras son: Fernández, Joseph A. (1982/1985). "The Allophones of lb, d, g/ in Costa Rican Spanish". Orbis 31, 1-2; 121-146 y López Guido, Flor de María. (1987). Análisis cuantitativo de las variantes alofónicas del fonema Id/ en el español del Área Metropolitana de San José: un enfoque sociolingüístico. Tesis de Maestría. Universidad de Costa Rica.

${ }^{14}$ Esta asimilación también puede involucrar a otras consonantes oclusivas como a las bilabiales, en casos como abuelo > agüelo.
} 
neutralización de /t/ > /p/ antes de /s/, se destaca la pérdida de la consonante oclusiva, bilabial, sorda, en posición de coda silábica. Este tipo de pérdidas son frecuentes en el habla popular por la tendencia a la sílaba abierta frente a la sílaba cerrada.

Para cerrar este apartado acerca de la fonética, falta comentar un ejemplo muy interesante. Se incluye en el polómetro dirigido a los literatos costarricenses (ver nota final vi):

[16] ¿Cree usted que un escritor costarricense debe decir 'hale' en lugar de 'jale'; Anótese 5 puntos. Pol-lit-2009

Pese a que la pregunta, en primera instancia, quiere indagar sobre el uso de un rubro léxico, salta en el ejemplo un tema de carácter fonético. En el marinerismo halar ${ }^{15}$, persiste la articulación a la manera del antiguo fonema $/ \mathrm{h} /$, donde no se produce la reducción al cero fonético de la norma actual hispánica. Queda claro por el planteamiento de la pregunta que la sanción no recae en la conservación de este rasgo propio del español medieval, sino todo lo contrario en la posible hipercorrección de un hablante que asume la articulación como ajena a la variedad costarricense. Aquí hay un cambio de giro: no se consideran polas las costumbres lingüísticas costarricenses, sino la imitación de otras normas, muy posiblemente la peninsular. Se ve aquí que hay una revaloración del dialecto costarricense. Esta perspectiva caracteriza, particularmente, a este polómetro, como se verá y se comentará con mayor amplitud en el siguiente apartado.

\subsection{Morfología}

\subsubsection{Formas de tratamiento y otros elementos}

Los polómetros también realizan una evaluación de este nivel lingüístico. La atención se concentra en las formas de tratamiento y su uso pragmático, amén de las consideraciones de la naturaleza idiosincrática propia del voseo para los costarricenses. Colateralmente, se discute sobre la "corrección" o "incorrección" de las desinencias verbales asociadas. No obstante, también se valoran otras partículas de este nivel, como el uso de las preposiciones.

\footnotetext{
${ }^{15}$ Voz proveniente de la palabra francesa haler, de acuerdo con el diccionario de la Real Academia Española.
} 
En cuanto a las formas de tratamiento, se parte de una premisa: usted es la forma consustancial a la manera de hablar costarricense. Así, en [17], está claramente expresado este punto de partida para evaluar la conducta lingüística de un hablante costarricense; es excluyente de cualquier otro pronombre usado como forma de tratamiento. Se sanciona tanto el uso de tú como de vos, aunque este último se ha asociado indefectiblemente con la identidad lingüística costarricense. Se trata de la defensa a ultranza del uso del pronombre usted como identificador de la variedad hispánica nacional:

[17] Usa tú o vos en vez de usted. Pol-2012

Por su parte, en el ejemplo [18] se acepta ahora el uso de vos como forma normal del uso idiomático costarricense, equiparándolo con el tratamiento de usted; es decir se amplía el repertorio de posibilidades. En todo caso, el principal objetivo de este ítem es la censura para el uso del pronombre tú. Incluso el redactor para ser congruente con su evaluación plantea la pregunta con la forma pronominal usted, pronombre que destaca con mayúsculas.

A diferencia de los resultados de Jara Murillo (2006, p. 76), donde la referencia al uso de las formas de tratamiento fue más bien baja; en los polómetros, por la aparición continuada en las diferentes versiones anuales, parece ser una preocupación manifiesta. La defensa de usted o vos, con preferencia por el primero, se convierte en una manifestación de lealtad lingüística a las costumbres idiomáticas costarricense:

[18] Habla UD de "tú", sabiendo que los ticos usamos el Vos o el Usted? Pol-sf

Esta actitud de defensa del voseo se expresa con vehemencia en [19]. Se castiga con una puntuación muy alta que ubicaría al encuestado fácilmente en la categoría de polo. Se equipara el voseo con un bien que ha de ser conservado, aquilatado y apreciado en grado sumo. Este ejemplo es una invitación a la conservación de un rasgo identitario y, por tanto, a la lealtad incondicional respecto del voseo:

[19] “¿Es usted costarricense y el voseo le vale mierda? ¡Anótese diez!” Pol-lit-2009 
La adopción de las formas del tuteo, tanto pronominales como verbales, son muestra de "policidad", es decir, son acreedoras de la sanción social y, por tanto, prototípicas de los hablantes cursis y afectados; sobre todo si se mezcla el tratamiento de vos y tú, como se documenta en el siguiente ejemplo:

[20] Habla de tú, pero falla en el intento. Si habla bien, no tiene nada de malo, pero..."Oye, qué malo sos". Pol-2015

No obstante, en [20], tímidamente hay una aceptación del tuteo. Este cambio de valoración es sintomático, ya que este ejemplo no surge hasta la versión del año 2015. Las investigaciones dialectológicas más recientes en nuestro país han detectado un avance en el uso del pronombre tú, al punto de pasar de un sistema bipolar a una triada pronominal. Al respecto, señala Miguel Ángel Quesada:

Al igual que en Bolivia [...], en Guatemala, El Salvador y Honduras y, en forma incipiente, en Costa Rica, se ha incrementado, al menos en el habla formal y entre las personas de alta escolaridad, el uso de tú, el cual se emplea para interlocutores desconocidos de la misma edad o con los mismos intereses, reservándose el voseo para el trato íntimo familiar y con los amigos más cercanos [...]. (Quesada Pacheco, M. Á., 2002, p. 110)

En todo caso, la sanción se dirige hacia la mezcla de los paradigmas correspondientes al voseo y al tuteo. Incluso, se ofrece un ejemplo de las construcciones que se deben evitar a toda costa: "Oye, qué malo sos", donde el primer verbo se conjuga siguiendo el modelo del tuteo y el segundo, el del voseo. La inconveniencia de este uso se enfatiza con recursos visuales, pues se ilustra con un fotograma del protagonista de la serie "House of cards", quien se caracteriza por sus aviesas intenciones en el mundo de la política estadounidense ${ }^{17}$.

Dentro de las apreciaciones sobre el uso de las formas de tratamiento, también hay referencias a datos del contexto de la comunicación, es decir a restricciones diafásicas para su uso.

\footnotetext{
${ }^{16}$ La versión del polómetro del año 2015 se publicó acompañada de ilustraciones (fotografías, diseños animados, memes, etc), con el fin de amenizar el contenido y, quizás, para clarificar los significados sugeridos.

17 "House of Cards", producción de la compañía Netflix, es una serie televisiva cuyo protagonista es el personaje Frank Underwood, político de aviesas intenciones en el mundo de la política estadounidense.
} 
En [21], se considera inapropiado el uso de "tú" en las relaciones amorosas. No obstante, este uso en situaciones románticas, según Vargas Dengo (1974, p. 29), fue asociado a los valores "normales” del tuteo en el español de Costa Rica. Curiosamente, la condena no se restringe solo a este contexto comunicativo, sino que se extiende a cualquier otra, pues con solo usar "tú" se acredita un doble puntaje en la medición de la condición de "polo":

[20] ¿Le habla usted a su novio o novia en "tú"? Anótese un punto. ¿Le habla a todo el mundo en "tú"? Anótese dos. Pol-lit-2009

En la misma versión del 2009, especializada para los escritores, se agrega un ítem para advertir cuán inapropiado es utilizar "tú" en la escritura de textos poéticos o narrativos. Aquí es indudable que se establece una restricción diafásica de uso:

[21] ¿Escribe usted poesía costarricense en 'tú'? Anótese un punto. ¿Escribe usted narrativa costarricense en 'tú'? Si la respuesta es afirmativa, sálgase de este blog: ya perdió todos los puntos. Pol-lit-2009 18

Asimismo, este ejemplo recuerda las tentativas decimonónicas de los intelectuales latinoamericanos por definir una variedad lingüística que expresara la identidad de sus pueblos recién liberados del dominio español. Por ejemplo, estos intentos trataron de reformar la ortografía castellana para reflejar las nuevas variedades hispánicas ${ }^{19}$.

\footnotetext{
${ }^{18}$ El planteamiento de la sanción se sale un poco de la lógica de los polómetros, porque en vez de perder todos los puntos, debería ganarse todos por usar tú en esos registros, con lo cual se adquiriría la condición de polo. Sin embargo, la expulsión por pérdida también puede interpretarse que no es necesaria la medición del polómetro para determinar que es un hablante de una variedad sancionada negativa por la comunidad de hablantes costarricenses.

${ }^{19}$ Es oportuno citar aquí las consideraciones de Guillermo Guitarte: "Se ve, pues, que posiblemente hasta mediados del siglo pasado la variedad solemne y elevada del habla de las capas sociales altas de Hispanoamérica solía utilizar la z. Persistía como norma culta la pronunciación de España, que en el lenguaje, como en todas las actividades, había dado la pauta de conducta en América durante los tres siglos de la época colonial. Por esto, el seseo, a pesar de reconocerse como universal en el Nuevo Mundo, era considerado un vicio o provincialismo que debía corregirse. Pronto, sin embargo, el grupo superior de los criollos se pasaría totalmente al partido americano en materia de lenguaje. El movimiento comenzó al generalizarse el romanticismo, que valorizó a través de la historia la forma de vida peculiar y llena de sentido de cada pueblo. A partir de él, los pensadores, artistas y literatos hispanoamericanos buscaron y descubrieron lo autóctono y natural, y reinvindicaron a lo criollo y le dieron un prestigio literario de que hasta entonces había carecido. Uno de los grandes escritores románticos hispanoamericanos, Domingo F. Sarmiento, propuso una reforma ortográfica para América en 1843, veinte años después de la iniciativa de García del Río y Bello" (La constitución de una norma del español general: el seseo, 1983, p. 103).
} 
Esta exhortación es extrema porque su incumplimiento provoca el ostracismo de la comunidad de participantes de un blog. Se podría pensar como una metáfora del costarricense polo que será excluido de su comunidad de habla.

\subsubsection{Desinencias verbales}

Las referencias de los polómetros a este aspecto de la morfología, se desarrollan paralelamente con las formas de tratamiento, expuestas en el apartado anterior. Se discute sobre la corrección o no del voseo desinencial, específicamente para el pretérito perfecto simple. En la mayoría de los casos, se agregan ejemplos de verbos conjugados. En Costa Rica, desde la primera investigación sistemática del voseo (Vargas Dengo, 1974, p. 24), se testimonia la presencia de este uso. En las versiones de los polómetros del 2005, 2008 y 2009 se repite la estructura presente en [22]:

[22] Le agrega 'eses' a todas las palabras, dijistes, prestastes, vinistes? Pol-2008, Pol-2015

Este fenómeno ha sido tratado en muchos manuales de dialectología hispanoamericana. Siempre se trata cuando se expone el voseo en América. Se ha explicado como una hipercorrección para los mismos voseantes, quienes examinan la conjugación del verbo siguiendo el paradigma del tuteo. Por otra parte, también se analiza como un proceso de disimilación. En una de las obras fundacionales de la nueva dialectología costarricense, Arturo Agüero esboza esta explicación:

No porque haya tendencia entre nosotros a cambiar el vos por el tú se dice vos cantaste, comiste, sentiste (2. a persona plural, el pronombre y $2 .{ }^{\text {a }}$ singular del verbo), sino porque ha influido la conjugación escolar en ello. Debe observarse que solo en estas formas verbales del pretérito sucede este fenómeno, no en las demás. Hay que aceptar, pues, que en la supresión de la $-s$ en la segunda persona plural del pretérito perfecto simple ha influido el haberse oído en la escuela y colegios de enseñanza media la conjugación de los verbos y, por consiguiente, la segunda persona singular solamente usada aquí en la lengua literaria, unido esto a un fenómeno de supresión (apócope en este caso) por disimilación, puesto que en estas formas verbales hay siempre dos eses (cantastes, comistes, salistes). Cualquiera de estas causas, o todas juntas, podrían considerarse, mas no que los hablantes costarricenses tiendan 
a usar las formas de la segunda persona singular, ni siquiera la del pretérito perfecto simple del indicativo. (Agüero Chaves, 2009, p. 93-94)

En la versión 2009-2010, se recupera este mismo ítem con la peculiaridad de que se ofrece un comentario enciclopédico, con el objeto de identificar al hablante "polo" con un género musical considerado de mal gusto, por algunos sectores de la sociedad nacional. En concreto, se dice que la presencia de voseo desinencial es propia de un cantante de reggaetón (Mr. Pray), cuyas canciones son programadas en la sección "La diosa del reggaetón", del programa televisivo conocido como "Intrusos de la farándula":

[23] Le agrega "eses" a todas las palabras, dijistes, prestastes, vinistes? Doble puntaje si no le importa porque asi [sic] habla Mr Pray el de la diosa del reggaetón. Pol-2009-2010

Por último sobre las formas de tratamiento, hay un comentario [24] que se acerca mucho a las consideraciones teóricas realizadas por los expertos naciones en dialectología. Se aclara el origen de la conjugación del voseo; se trata, pues, de una aclaración etimológica del voseo desinencial. Aquí se cambia la perspectiva, pues se considera polo lo que en las otras versiones se sanciona: la utilización de las desinencias correspondientes a tú en los verbos conjugados en pretérito perfecto simple. Entonces, este comentario se convierte en una defensa del uso popular que coincide con la explicación diacrónica del fenómeno. Este cambio de interpretación de los rasgos vitandos, quizás, se debe a que el autor de esta versión es un escritor costarricense que ha adquirido conocimientos teóricos de la variación dialectal hispánica en América y, en particular, en Costa Rica:

[24] ¡Cree usted que usar una "s" en los verbos de segunda persona del singular es polo? (Por ejemplo: tuvistes; vinistes). Anótese un punto. ¿Ignoraba usted que esa "s" corresponde a la conjugación clásica del voseo y que por tanto es correcta en los países donde se vosea? Anótese dos. Pol-lit-200 


\subsubsection{Conjunciones}

Los polómetros no son pródigos en menciones a otras clases de palabras. Sin embargo, hay una referencia constante al uso de las conjunciones adversativas y a los pronombres posesivos. En ambos casos, se les considera pleonasmos. Llama la atención que se utilice un término del metalenguaje gramatical. Este empleo de un tecnicismo demuestra la actitud prescriptivista. Se trata de un apoyo desde la autoridad para sancionar un uso popular del lenguaje. Se ofrece un ejemplo presente en las versiones del 2005, 2008, 2008-reloaded y 2009-2010:

[25] Utiliza pleonasmos tales como "mas sin embargo" y "su carro de él”? Pol-2005, Pol2008, Pol-2008 reloaded y Pol-2009-2010

En cuanto a las conjunciones, la construcción considerada viciosa es "mas sin embargo", la cual es muy frecuente con su equivalente "pero sin embargo" en el habla costarricense, donde puede llegar a ser usada incluso por hablantes de la norma culta costarricense.

Por su parte, se sanciona el uso simultáneo de la forma sintética del pronombre posesivo "su" y de la forma analítica “de él”. Se sabe que estas construcciones pueden presentarse en el sistema de posesivos del español atlántico, con el afán de desambiguar el significado polisémico de "su"20.

\footnotetext{
${ }^{20}$ Para más detalles sobre este fenómeno, se puede consultar: Granda, G. de. (1978). "La evolución del sistema de posesivos en el español atlántico". En: Estudios lingüísticos hispánicos, afrohispánicos y criollos. Madrid: Gredos, 89-94.
} 


\section{Conclusiones}

Este estudio partió del análisis de la definición de polo, -a, presente en el Nuevo Diccionario de Costarriqueñismos de Miguel Ángel Quesada. Del examen de la acepción segunda, se postuló la existencia de un tipo social caracterizado por su mal gusto, baja escolaridad, etc. Se constató que los polómetros son textos excelentes para conocer estas características y, en especial, la variedad lingüística que se asocia a los hablantes excluidos socialmente. Así se completó este perfil sociolingüístico.

Ahora para concluir, es preciso reproducir la definición de "politico", como grado último de “policidad" que se incluye en la versión de 2012:

[26] De 31 a 40 puntos: Politico. Pregúntele a Dios que hizo usted para merecer semejante castigo. No es de raro que le guste el reggaeton y que tenga el cuarto decorado con posters de Bob Esponja o Las Chicas Superpoderosas. La Extra es su periódico favorito y se cree todas las varas que dicen sobre Extraterrestres y otras tonteras. Pida un milagro y si no se lo conceden, resígnese a vivir así, que le queda? Pol-2012

La censura social del polo se refuerza con la referencia al plano religioso. Es decir, asumir una conducta pola, incluido el uso del lenguaje asociado, es casi una condena divina; con lo cual queda demostrada la extrema programación social a la que nos encontramos como integrantes de una sociedad y, en específico, de una comunidad de habla.

Todas las versiones encontradas, excepto la dirigida a escritores literarios costarricenses, apoyan esta condena y muestran en detalle el perfil social que debe evitarse. En cuanto a la versión dirigida a literatos, esta es una reacción a esa configuración social; con respecto a la valoración lingüística defiende el cambio de la lengua; en otras palabras, se acerca más a la posición de la 
dialectología académica. Su concepto de policidad consiste más bien en la aceptación de esa construcción social excluyente.

\section{Bibliografía}

Agüero Chaves, A. (1996). Diccionario de Costarriqueñismos. San José: Sección de Publicaciones y Extensión Cultural de la Biblioteca Monseñor Dr. Víctor Manuel Sanabria de la Asamblea Legislativa.

Agüero Chaves, A. (2009). El español de Costa Rica. San José: Editorial UCR.

Alvarado, Ó. (s.f.). www.angelfire.com. Recuperado de http://www.angelfire.com/ky3/ozweb/polometro.html Consulta: 26 de julio de 2015.

Anónimo. (7 de enero de 2010). www.ahweon.blogspot.com. Recuperado de http://ahweon.blogspot.com/2010/01/polometro-2009-2010.html Consulta: 27 de julio de 2015.

Anónimo. (2015). www.facebook.com. Recuperado de https://www.facebook.com/puravidacrc/posts/326064557478504 Consulta: 27 de julio de 2015.

Calvo Shadid, A. (1995). Variación fonética de /r/ y /r/ en el habla culta de San José. Revista de Filología y Lingûistica, XXI (1), 115-134.

Calvo Shadid, A. (2008). Las semiconsonantes y semivocales en los diptongos del español: propuesta de análisis fonológico. Revista de Filología y Lingüística, XXXIV (2), 107-142.

Chakra. (11 de enero de 2007). www.forodecostarica.com. Recuperado de http://www.forodecostarica.com/discusion-general/354-polometro.html Consulta: 27 de julio de 2015 .

Chiri. (diciembre de 2004). www.basociacin4x4extremocostarica.runboard.com. Recuperado de http://basociacin4x4extremocostarica.runboard.com/t1379 Consulta: 27 de julio de 2015.

Cros, E. (2003). El sujeto cultural, sociocrítica y psicoanálisis. Medellín: Fondo Editorial Universidad EAFIT.

Flores Cornejo, J. C. (2012). Del "polo" a lo "polo": insinuaciones pragmáticas. Herencia, 25 (1 y 2), 107-115.

Gagini, C. (2008). Diccionario de Costarriqueñismos. San José. C R: Editorial Costa Rica.

Godínez, L. (2013). Actitudes lingüísticas hacia el habla campesina en el cantón de Pérez Zeledón. San José. 
Granda (de), G. (1978). La evolución del sistema de posesivos en el español atlántico. En G. Granda (de), Estudios lingüísticos hispánicos, afrohispánicos y criollos. Madrid: Gredos, 89-94.

Guitarte, G. (1983). La constitución de una norma del español general: el seseo. En G. Guitarte, Siete estudios sobre el español de América. México: UNAM, 99-106

Haensch, G. (1982). La lexicografía. De la lingüística teórica a la lexicografía práctica. Madrid: Gredos S. A.

Jaén, X. (mayo de 1994). El habla como un medio de movilidad social. Revista Comunicación, 8 (2), 69-74.

Jara Murillo, C. (2006). El español de Costa Rica según los ticos: un estudio de lingüística popular. San José: Editorial UCR.

Moreno Fernández, F. (1998). Principios de sociolingüística y sociología del lenguaje. Barcelona: Ariel Lingüística.

Niedzielki, N., \& Preston, D. (2003). Folk Linguistics. Berlin/ New York: Mouton de Gruyter.

Obando, A. (12 de octubre de 2009). www.elmasviolentoparaiso.blogspot.com. Recuperado de http://elmasviolentoparaiso.blogspot.com/2009/10/polometro-para-escritores.html Consulta: 27 de julio de 2015.

Quesada Pacheco, M. (1996). Los fonemas del español de Costa Rica. Aproximación dialectologica. Lexis, XX (1-2), 535-562.

Quesada Pacheco, M. Á. (1989). Actitudes hacia el habla campesina de Costa Rica a través de su historia. Herencia, 1 (2), 72-82.

Quesada Pacheco, M. Á. (1996). El español de América Central. En M. Alvar, Manual de dialectología hispánica. El español de América. Barcelona: Editorial Ariel S. A., 101-115.

Quesada Pacheco, M. Á. (2002). El Español de América (2. ${ }^{\text {a }}$ ed.). Cartago: Editorial Tecnológica de Costa Rica.

Quesada Pacheco, M. Á. (2007). Nuevo Diccionario de Costarriqueñismos (4. a ed.). Cartago: Editorial Tecnológica de Costa Rica.

Quesada Pacheco, M. Á. (2007). Nuevo Diccionario de Costarriqueñismos. Cartago: Editorial Tecnológica de Costa Rica.

Rivera Villanueva, R. (5 de enero de 2015). www.medium.com. Recuperado de https://medium.com/@Rafalogo/polometro-2015-9f527b216b1a Consulta: 19 de mayo de 2015. 
Káñina, Rev. Artes y Letras, Univ. de Costa Rica XLII (1) 2018: 145-173 /ISSN: 2215-2636

Romeovf. (30 de junio de 2008). www.forodecostarica.com. Recuperado de http://www.forodecostarica.com/solaz/58039-polometro-2011-alguien-lo-tiene.html Consulta: 27 de julio de 2015.

Vargas Dengo, C. (1974). El uso de los pronombres "vos" y "usted" en Costa Rica. Revista de Ciencias Sociales de la Universidad de Costa Rica, (88), 7-30.

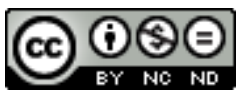

Esta obra está bajo una licencia de Creative Commons Reconocimiento-NoComercial-SinObraDerivada 4.0 Internacional. 